\section{Fecal calprotectin correlates well with endoscopic activity in ulcerative colitis patients}

\section{To the Editor,}

We read with interest the meta-analysis published by Rokkas et al. [1] about the diagnostic performance of fecal calprotectin (FC) in assessing inflammatory bowel disease (IBD) endoscopic activity in adults. In view of the clinical management of UC, it is essential to determine the disease activity. At present, the most accurate way to evaluate the severity of UC is endoscopy with biopsy. However, this technique has the drawbacks of being invasive, timeconsuming, burdensome to the patient and expensive. That is why noninvasive biomarkers such as FC are more frequently used for monitoring IBD activity [2]. There are several different scoring systems for the endoscopic evaluation of UC severity, such as endoscopic Mayo score (EMS), modified Baron score, etc. However, none of these instruments have been validated for a reliable assessment [3]. More recently, the Ulcerative Colitis Endoscopic Index of Severity (UCEIS) has been reported by Travis et al. [4]. This score was developed as an index that captures $90 \%$ of the variance in the overall assessment of endoscopic severity.

We would like to present the experience of our Bulgarian center regarding the correlation between $\mathrm{FC}$ and the endoscopic activity in ulcerative colitis (UC) patients, assessed by UCEIS score and EMS.

Enrolled were 116 consecutive UC patients (52 females; mean age $38.9 \pm 9$, range $18-63$; 56 with quiescent UC, 60 with active UC) and 36 controls. Endoscopies were performed by two experienced board-certified gastroenterologists who graded the findings according to EMS and UCEIS. EMS 0 was accepted as remission. Fecal calprotectin was analyzed in stool samples by means of point-of-care desk-top Quantum Blue (POC) method.

There was no statistically significant difference between mean FC levels in controls $(34.72 \pm 7.43 \mu \mathrm{g} / \mathrm{g})$ and UC patients in remission $(47.10 \pm 26.91 \mu \mathrm{g} / \mathrm{g})(\mathrm{p}=0.205)$. However, the patients with active UC had significantly higher mean FC levels $(1933.08 \pm 940.98 \mu \mathrm{g} / \mathrm{g})$ than controls $(\mathrm{p}<0.001)$ and patients with quiescent UC $(\mathrm{p}<0.001)$. Fecal calprotectin level significantly correlated with UCEIS $(r=0.869)$ and EMS ( $r=0.814$ ) (Fig.1). For both items, $\mathrm{p}<0.001$ was found. Moreover, UCEIS had very strong correlation with EMS ( $r$ $=0.922, \mathrm{p}<0.001)$. This prospective pilot study also showed a good correlation between FC and endoscopic activity in UC.

In our group of quiescent UC, the highest FCP value was $136 \mu \mathrm{g} / \mathrm{g}$. On the other hand, FCP concentrations in patients with active UC were significantly higher than those of controls and patients in remission so that we could not detect a cut-off level between activity and remission in UC patients. Rokkas et al [1] studied three FC cut off levels, i.e. FC up to $50 \mu \mathrm{g} / \mathrm{g}$, FC up to $100 \mu \mathrm{g} / \mathrm{g}$ and $\mathrm{FC}>100 \mu \mathrm{g} / \mathrm{g}$ and showed that the higher the cut-off was, the lower was the sensitivity, but the higher was the specificity. This means that FC test showed its best performance (sensitivity $90.6 \%$, AUC 0.91 ) at the cut-off level of $50 \mu \mathrm{g} / \mathrm{g}$. In our opinion this cut off level is too low and will lead to many unnecessary colonoscopies or treatment escalation. We are wondering whether a higher cut off, i.e. 150 $\mu \mathrm{g} / \mathrm{g}$ will be more useful in clinical practice both in terms of diagnosis and monitoring.

As pointed by previous studies, FC can be used as an alternative to endoscopy and can be accepted as a direct biomarker of intestinal inflammation in UC patients [1, 4]. It is less expensive and non-invasive compared to colonoscopy. Our results underscore the importance of FC in evaluation of disease activity in UC on daily practice.

In this study FC was analyzed by means of POC method. We find this test really useful, because it is simple to use, can be done in doctor's office and is quite fast (results can be obtained in less than $30 \mathrm{~min}$ including protein extraction). Another major advantage is the simplicity of sample preparation and analysis. No more than $80 \mathrm{mg}$ stool sample is required for the assessment and sample preparation and analysis is user friendly and does not involve the need of special equipment, which makes it ideally suited for every lab and even doctor's 


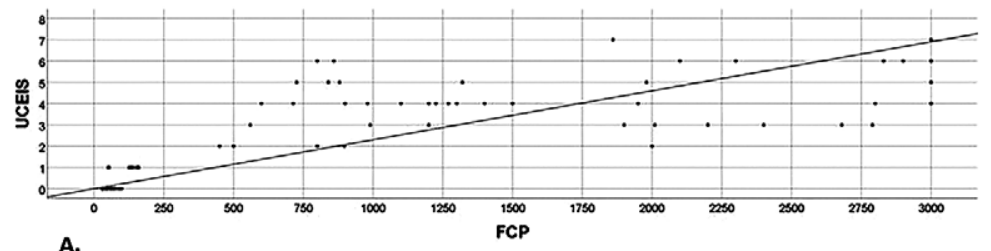

A.

FCP

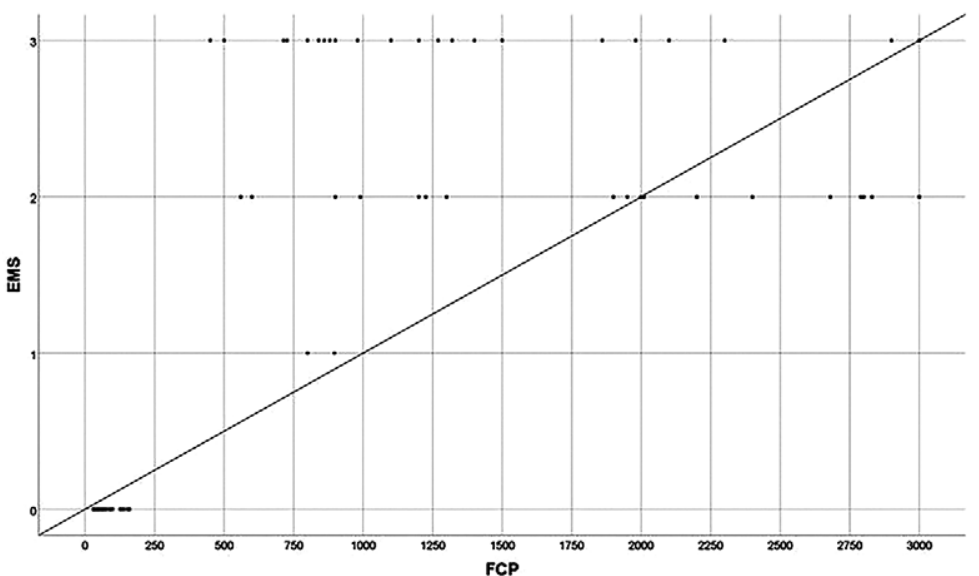

Fig. 1. Correlation between fecal calprotectin (FCP) concentration and A. Ulcerative Colitis Endoscopic Index of Severity (UCEIS) $(r=0.869)$ and B. Mayo endoscopic score (EMS) $(r=0.814)$. Spearman's correlation test.

office. Moreover, the point-of-care test can serve as a reliable alternative to ELISA $[5,6]$. It has been shown that POC method is the instrument of choice for fast and reliable determination of FCP levels.

In conclusion, FC concentration demonstrated good correlation with endoscopic activity in UC according to UCEIS and EMS. Our study provides further evidence that FC represents a useful biomarker for noninvasive monitoring of disease activity in UC patients.

\section{Radislav Nakov, Ventsislav Nakov, Vanya Gerova, Lyudmila Tankova}

Clinic of Gastroenterology, "Tsaritsa Yoanna” University Hospital, Medical University of Sofia, Sofia, Bulgaria

Correspondence: Radislav Nakov, radislav.nakov@gmail.com

Conflicts of interest: None.

DOI: 10.15403/jgld.2014.1121.274.cal

\section{REFERENCES}

1. Rokkas T, Portincasa P, Koutroubakis IE. Fecal calprotectin in assessing inflammatory bowel disease endoscopic activity: a diagnostic accuracy meta-analysis. J Gastrointestin Liver Dis 2018;27:299-306. doi:10.15403/ jgld.2014.1121.273.pti

2. Lewis JD. The utility of biomarkers in the diagnosis and therapy of inflammatory bowel disease. Gastroenterolgy 2011;140:1817-1826. doi:10.1053/j.gastro.2010.11.058

3. D'Haens G, Sandborn WJ, Feagan BG, et al. A review of activity indices and efficacy end points for clinical trials of medical therapy in adults with ulcerative colitis. Gastroenterology 2007;132:763-786. doi:10.1053/j.gastro.2006.12.038
4. Travis SP, Schnell D, Krzeski P, et al. Developing an instrument to assess the endoscopic severity of ulcerative colitis: the Ulcerative Colitis Endoscopic Index of Severity (UCEIS). Gut 2012;61:535-542. doi:10.1136/gutjnl-2011-300486

5. Lee YW, Lee KM, Lee JM, et al. The usefulness of fecal calprotectin in assessing inflammatory bowel disease activity. Korean J Intern Med 2018 Jan 20. doi:10.3904/kjim.2016.324

6. Coorevits L, Baert FJ, Vanpoucke HJ. Faecal calprotectin: comparative study of the Quantum Blue rapid test and an established ELISA method. Clin Chem Lab Med 2013;51:825-831. doi:10.1515/cclm-2012-0386

\section{Reply,}

\section{To the Editor,}

We thank Nakov R. et al. for their remarks on the correlation of fecal calprotectin (FC) with endoscopic activity in Ulcerative Colitis (UC) patients. We are pleased that our meta-analysis "Fecal calprotectin in assessing inflammatory bowel disease endoscopic activity: a diagnostic accuracy meta-analysis" [1] prompted them to analyze their data. Their results showed that in UC patients FC concentration has a good correlation with two endoscopic activity scores, i.e. Ulcerative Colitis Index Score (UCEIS) and Endoscopic Mayo Score (EMS). They concluded that their results provide further evidence that FC represents a useful biomarker for noninvasive monitoring of disease activity in UC patients. Their conclusion is in accordance with the conclusion of our meta-analysis and indeed stresses the usefulness of FC, as a noninvasive biomarker, in assessing inflammatory bowel disease endoscopic activity. Concerning the cut-off levels which show optimal accuracy, our meta-analysis showed a level of 50 
$\mu \mathrm{g} / \mathrm{g}$. Nakov et al. wonder whether the use of a higher cut-off level of FC would not be better for preventing unnecessary colonoscopies or treatment escalation. We agree that in the future, more relevant studies might change this level, as will be shown with updated meta-analyzes. From this point of view, we would like to encourage them to enlarge this pilot study and then publish their interesting data.

Theodore Rokkas ${ }^{1}$, Piero Portincasa ${ }^{2}$, Ioannis E. Koutroubakis ${ }^{3}$ 1) Department of Gastroenterology, Henry Durant Hospital Center, Athens, Greece; 2) Division of Internal Medicine, „Aldo Moro” University, Bari Medical School, Bari, Italy; 3) Department of Gastroenterology, University Hospital, Heraklion, Crete, Greece

Correspondence: Theodore Rokkas, sakkor@otenet.gr

Conflicts of interest: None.

DOI: $10.15403 /$ jgld.2014.1121.274.ply

\section{REFERENCES}

1. Rokkas T, Portincasa P and Koutroubakis IE. Fecal calprotectin in assessing inflammatory bowel disease endoscopic activity: a diagnostic accuracy meta-analysis. J Gastrointestin Liver Dis 2018;27:299-306. doi:10.15403/jgld.2014.1121.273.pti

\section{Bougie cap access for successful intubation of the upper esophageal sphincter in diffuse idiopathic skeletal hyperostosis-related dysphagia („DISHphagia“)}

\section{To the Editor,}

An esophago-gastro-duodenoscopy (EGD) is usually a straightforward and easy-to-accomplish endoscopic procedure. However, difficulties in accessing the esophagus may occur in a minority of patients, and have recently been addressed by Siau K. et al. [1]. While in clinical practice termination of the procedure with subsequent radiography may be an option, I present another option to proceed with endoscopy designated "bougie cap access".

This is illustrated in an elderly patient presenting with cervical dysphagia for solids. While routine ultra-slim EGD failed (Fig. 1A), an upper gastrointestinal study indicated filiform contrast passage through the sphincter with exuberant adjacent bridging osteophyte formation. Advanced diffuse idiopathic skeletal hyperostosis (DISH) was confirmed by cervical spine computed tomography ("DISHphagia") (Fig. 1B) [2]. The patient underwent repeat EGD using a pediatric endoscope with an attached bougie cap device. Fig. 2 shows the tight contact with markedly improved endoscopic visualization of the tight upper esophageal sphincter (UES) passed easily by minimal pressure, excluding mucosal pathology within the strictured segment. The therapeutic options were discussed with the patient beforehand, who was considered to be a poor candidate for surgical decompression due to advanced age and significant pulmonary co-morbidity [3]. Therefore, in light of insufficient evidence and low clinical rationale for a therapeutic
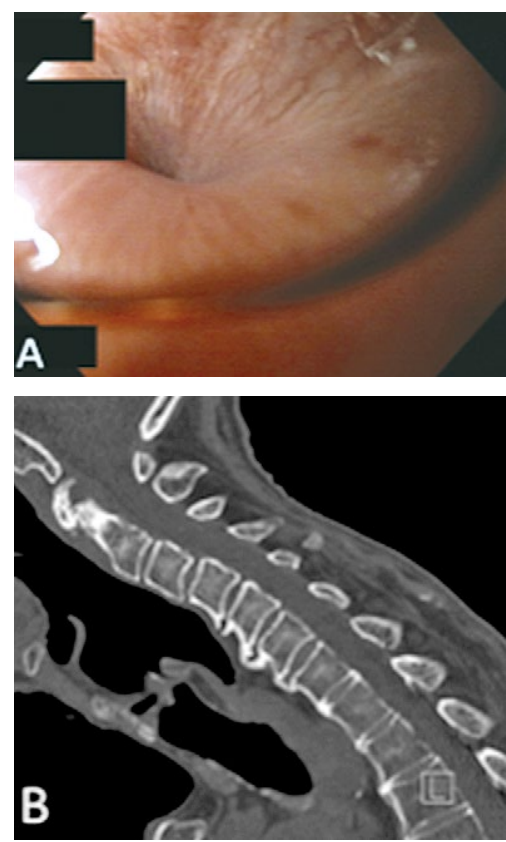

Fig. 1. (A) Endoscopic illustration of the stricture at the level of the upper esophageal sphincter, precluding intubation with a pediatric endoscope (GIF XP180, Olympus Hamburg, Germany). (B) Cervical computed tomography in the sagittal plane indicating bridging osteophyte formation at the corresponding level.

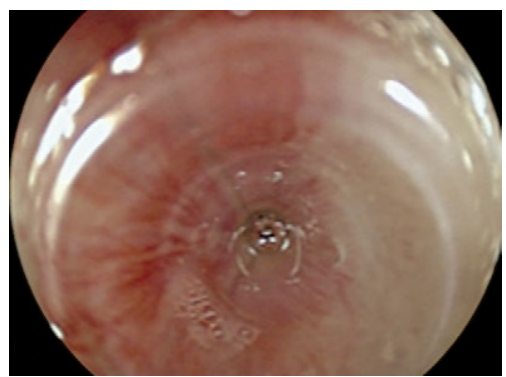

Fig. 2. Firm alignment into the tight upper esophageal sphincter exhibiting focal mucosal whitening and vessel injection. Endoscopic passage is achieved by minimal pressure with the remaining esophagus being unremarkable.

bougienage trial, the patient opted for definitive continuation of soft food intake with adjunctive enteral nutrition provided over a (push-type) percutaneous endoscopy gastrostomy.

The single-use BougieCap (Ovesco, Tübingen, Germany) has recently been marketed in Europe for esophageal bougienage with or without a guidewire. As a dome-shaped modification of the traditional cap design, the BougieCap is attached to the scope tip and is available in sizes up to $16 \mathrm{~mm}$ (20 $\mathrm{mm}$ in the future). While unique clinical applications, e.g. as a tool for calibration of lumen diameter in eosinophilic esophagitis have been reported, more rigorous testing of its utility in clinical endoscopy is under way $[4,5]$. The safety of this new esophageal bougienage technique has formally not been established. However, in regard of the additional visual 
control of the extent of tissue tension relative to e.g. SavaryMiller bougienage, perforation and/or bleeding risks might be reduced, albeit up to now no systematic data and/or headto-head comparative trials have been reported. Nevertheless, as is illustrated herein, with the conical cap design, strictures might be navigated under full endoscopic control and the force axis is stabilized by the tip of the cap aligning into the stricture. This is in contrast to traditional cap-assisted endoscopy, albeit standard-type caps being commercially unavailable for ultraslim endoscopes.

In conclusion, bougie cap-assisted EGD might be an attractive novel concept in cervical pathologies, including e.g. cricopharyngeal bar, Plummer-Vinson-associated strictures and/or webs, with improved endoscopic assessment of naïve mucosal pathology distinguishing the procedure from e.g. endoscopic access after limited fluoroscopy and guidewiredirected dilation/bougienage.

\section{Vincent Zimmer}

Department of Medicine, Marienhausklinik St. Josef Kohlhof, Neunkirchen and Department of Medicine II, Saarland University Medical Center, Saarland University, Homburg, Germany

Correspondence: Vincent Zimmer, vincent.zimmer@gmx.de

Conflicts of interest: None.

DOI: $10.15403 /$ jgld.2014.1121.274.dys

\section{REFERENCES}

1. Siau K, Li J, Fisher NC, Mulder CJJ, Ishaq S. Intubation failure during gastroscopy: incidence, predictors and follow-up findings. J Gastrointestin Liver Dis 2017;26:339-344. doi:10.15403/ jgld.2014.1121.264.isq

2. Clark E, Preston P, Wates A, Merry P. DISHphagia--a difficult problem to swallow. Rheumatology (Oxford) 2003;42:1422-1423. doi:10.1093/ rheumatology/keg353

3. Bacigaluppi S, Merciadri P, Secci F, Bragazzi NL, Zona G. An unusual cause of dysphagia: „DISHphagia“. Br J Neurosurg 2015;29:275-276. do i:10.3109/02688697.2014.977777

4. Zimmer V. Bougie cap esophageal passage: A novel potential tool for calibration of lumen diameter in eosinophilic esophagitis? Gastrointest Endosc 2018;88:568-569. doi:10.1016/j.gie.2018.03.027

5. Walter B, Schmidbaur S, Hann A, Meining A. The bougie-cap: a new method for treatment of oesophageal strictures. Endoscopy 2018;50:S31. doi:10.1055/s-0038-1637117

\section{No changes in Interleukin-10 expression in symptomatic uncomplicated diverticular disease of the colon}

\section{To the Editor,}

Symptomatic uncomplicated diverticular disease (SUDD) is a common condition that affects about $25 \%$ of patients with colonic diverticulosis [1]. Although the pathogenesis is not completely understood, it is generally thought that a combination of microbial imbalance and low-grade inflammation are two milestones in the occurrence of symptoms [1]. Interleukin-10 (IL-10) is an important antiinflammatory mediator and suppresses the production of proinflammatory cytokines [2]. Since changes in some cytokines expression have been already shown in SUDD [3], the aim of the present study was to assess IL-10 expression in SUDD patients and to compare it to its expression in subjects with asymptomatic diverticulosis (AD) and healthy controls (HC).

We retrospectively assessed IL-10 expression in fecal samples of a population of 44 consecutive patients having the following characteristics: middle-aged female patients, birth by vaginal delivery and with exclusive breastfeeding, living in the same geographical area, similar body mass index (BMI), no use of antibiotics in the three months before enrolment, no ongoing or past acute complicated and uncomplicated diverticulitis; absence of bacterial and/or parasitic intestinal diseases and lactose malabsorption. We enrolled 15 SUDD patients, 13 patients with $\mathrm{AD}$, and $16 \mathrm{HC}$. SUDD was defined as the presence of symptoms in patients with diverticulosis detected by complete colonoscopy, with presence of abdominal pain recorded in the lower left quadrant for more than 24 hours and in the absence of any complication (stenosis, abscesses, fistulas) [4]. Stool samples were obtained at least 4 weeks after colonoscopy.They were collected early in the morning at home, in sterile plastic tubes, transported on ice to the laboratory within $2 \mathrm{~h}$ and stored at $-80^{\circ} \mathrm{C}$ until analysis. Enzymelinked immunosorbent assay (ELISA) for IL-10 (Human IL-10 Quantikine immunoenzymatic kit, R\&D Systems Inc., Minneapolis, MN, USA) was carried out on fecal samples according to the manufacturer's protocol, and expressed as picograms/gram (pg/g) of faeces. One-way analysis of variance was used to test the difference in mean values of IL-10 among the three study groups. The characteristics of the study groups were similar. The median age of the SUDD population was 64 (range 52-70) years, and the median BMI was 25.6 (range 21.6-33.7), with no differences between SUDD, AD and HC. With the exception of abdominal pain, the most frequent symptom was abdominal bloating, occurring in 10 patients (76.9\%). An increased release of IL-10 from HC controls up to $\mathrm{AD}$ and SUDD patients was observed. In particular, mean IL-10 expression was 3.32 (95\% CI 0.43-7.91) pg/g in HC, 3.42 (95\% CI 1.87-5.10) pg/g in AD and 4.24 (95\% CI 1.90-7.53) $\mathrm{pg} / \mathrm{g}$ in SUDD. However, no significant difference was found among the 3 study groups ( $\mathrm{p}=0.326$ ).

Decreased levels of IL-10 may predispose to increased proinflammatory cytokine production, contributing to a pattern of unbalanced cytokine profile in a subclinical inflammatory state. In SUDD, imbalance of some cytokines has been already detected. In particular, Tumor Necrosis Factor-alpha (TNF- $\alpha$ ) was found increased in colonic mucosa of those people [3]. We expected to find similar results in SUDD. We did not find any significant changes in the IL-10 expression between the studied groups, recording a slight increase, not statistically significant, from $\mathrm{HC}$ up to AD and SUDD. These results are similar to those recently reported by Turco et al. [5], who observed a progressive increase of IL-10 release in HC, AD and SUDD patients, although IL-10 release was significantly greater than 
HC only in SUDD patients with previous acute diverticulitis. Thus, we can speculate that IL-10 expression in SUDD may be an attempt of the immune system to control a low-grade inflammation, due to a change in colonic microbiota. Colonic microbiota imbalance can occur in those patients [6], triggering a low-grade inflammation and consequent symptoms [7]. This hypothesis seems to be confirmed by Dai et al. [8], who found an inverse expression of prostaglandin E2 in colonic mucosa of acute diverticulitis and inflammatory bowel diseases. We know that prostaglandin E2 is the dominant prostaglandin in the colon and it is associated with colonic inflammation, but it also has a protective mechanism in the mucosa of the gastrointestinal tract. A lowered prostaglandin E2 level may decrease the normal protection of the mucosa, which is made more susceptible to luminal insults, thus creating a permissive environment for the development of acute diverticulitis. The IL-10 behavior in SUDD may be further support to the hypothesis that low-grade inflammation could be the source for symptoms' occurrence in SUDD patients.

\section{Antonio Tursi ${ }^{1}$, Paola Mastromarino ${ }^{2}$, Daniela Capobianco ${ }^{2}$, Walter Elisei $^{3}$, Marcello Picchio ${ }^{4}$, Giovanni Brandimarte ${ }^{5}$ \\ 1) Gastroenterology Service, ASL BAT, Andria (BT); 2) Department of Public Health and Infectious Diseases, Section of Microbiology, Sapienza University, Rome; 3) Division of Gastroenterology, ASL RM6, Albano Laziale, Rome; 4) Division of Surgery, "P. Colombo" Hospital, ASL RM6, Velletri, Rome; 5) Division of Gastroenterology, "Cristo Re" Hospital, Rome, Italy}

Correspondence: Antonio Tursi, antotursi@tiscali.it

Conflicts of interest: None.

DOI: $10.15403 /$ jgld.2014.1121.274.ink

\section{REFERENCES}

1. Strate LL, Modi R, Cohen E, Spiegel BM. Diverticular disease as a chronic illness: evolving epidemiologic and clinical insights. Am J Gastroenterol 2012;107:1486-1493. doi:10.1038/ajg.2012.194

2. Neurath MF. Cytokines in inflammatory bowel disease. Nat Rev Immunol 2014;14:329-342. doi:10.1038/nri3661

3. Humes DJ, Simpson J, Smith J, et al. Visceral hypersensitivity in symptomatic diverticular disease and the role of neuropeptides and low grade inflammation. Neurogastroenterol Motil 2012;24:318-e163. doi:10.1111/j.1365-2982.2011.01863.x

4. Tursi A, Elisei W, Picchio M, Giorgetti GM, Brandimarte G. Moderate to severe and prolonged left lower-abdominal pain is the best symptom characterizing symptomatic uncomplicated diverticular disease of the colon: a comparison with fecal calprotectin in clinical setting. J Clin Gastroenterol 2015;49:218-221. doi:10.1097/MCG.0000000000000094

5. Turco F, Andreozzi P, Palumbo I, et al. Bacterial stimuli activate nitric oxide colonic mucosal production in diverticular disease. Protective effects of L. casei DG ${ }^{\circledast}$ (Lactobacillus paracasei CNCM I-1572). United European Gastroenterol J 2017;5:715-724. doi:10.1177/2050640616684398

6. Tursi A, Mastromarino P, Capobianco D, et al. Assessment of fecal microbiota and fecal metabolome in symptomatic uncomplicated diverticular disease of the colon. J Clin Gastroenterol 2016;50 (Suppl 1):S9-S12. doi:10.1097/MCG.0000000000000626

7. Kvasnovsky CL, Leong LEX, Choo JM, et al. Clinical and symptom scores are significantly correlated with fecal microbiota features in patients with symptomatic uncomplicated diverticular disease: a pilot study. Eur J Gastroenterol Hepatol 2018;30:107-112. doi:10.1097/ MEG.0000000000000995

8. Dai L, King DW, Perera DS, Lubowski DZ, Burcher E, Liu L. Inverse expression of prostaglandin E2-related enzymes highlights differences between diverticulitis and inflammatory bowel disease. Dig Dis Sci 2015;60:1236-1246. doi:10.1007/s10620-014-3478-7 